\title{
The Stokes-Einstein relation at moderate Schmidt number
}

\author{
Florencio Balboa Usabiaga, ${ }^{1}$ Xiaoyi Xie, ${ }^{2}$ Rafael Delgado-Buscalioni, ${ }^{1}$ \\ and Aleksandar Donev ${ }^{3, a)}$ \\ ${ }^{1}$ Departamento de Física Teórica de la Materia Condensada and IFIMAC Univeridad Autónoma de Madrid, \\ Madrid 28049, Spain \\ ${ }^{2}$ Department of Physics, New York University, New York, New York 10012, USA \\ ${ }^{3}$ Courant Institute of Mathematical Sciences, New York University, New York, New York 10012, USA
}

(Received 27 September 2013; accepted 12 November 2013; published online 5 December 2013)

\begin{abstract}
The Stokes-Einstein relation for the self-diffusion coefficient of a spherical particle suspended in an incompressible fluid is an asymptotic result in the limit of large Schmidt number, that is, when momentum diffuses much faster than the particle. When the Schmidt number is moderate, which happens in most particle methods for hydrodynamics, deviations from the Stokes-Einstein prediction are expected. We study these corrections computationally using a recently developed minimally resolved method for coupling particles to an incompressible fluctuating fluid in both two and three dimensions. We find that for moderate Schmidt numbers the diffusion coefficient is reduced relative to the Stokes-Einstein prediction by an amount inversely proportional to the Schmidt number in both two and three dimensions. We find, however, that the Einstein formula is obeyed at all Schmidt numbers, consistent with linear response theory. The mismatch arises because thermal fluctuations affect the drag coefficient for a particle due to the nonlinear nature of the fluid-particle coupling. The numerical data are in good agreement with an approximate self-consistent theory, which can be used to estimate finite-Schmidt number corrections in a variety of methods. Our results indicate that the corrections to the Stokes-Einstein formula come primarily from the fact that the particle itself diffuses together with the momentum. Our study separates effects coming from corrections to no-slip hydrodynamics from those of finite separation of time scales, allowing for a better understanding of widely observed deviations from the Stokes-Einstein prediction in particle methods such as molecular dynamics. ( 2013 AIP Publishing LLC. [http://dx.doi.org/10.1063/1.4834696]
\end{abstract}

\section{INTRODUCTION}

The self-diffusion coefficient $\chi$ of a tracer particle suspended in a liquid (for example, a colloidal particle) is a quantity of fundamental interest, and can be predicted using the well-known Stokes-Einstein (SE) relation. This famous formula is a combination of two results. The first, by Einstein, comes from rather general linear response theory considerations and relates the diffusion coefficient to the mobility $\mu, \chi$ $=k_{B} T \mu$ with $k_{B} T$ the thermal energy scale, where the mobility is defined by measuring the average steady velocity $\langle u\rangle$ of the particle in response to a weak applied force $F$, $\mu=\lim _{F \rightarrow 0}\langle u\rangle / F$. The second part of the SE relation is the Stokes formula for the mobility of a sphere suspended in a viscous fluid, obtained using standard hydrodynamics. While there is little reason to doubt the applicability of Einstein's relation, the Stokes-Einstein relation assumes that the drag force on a particle is not affected by thermal fluctuations, that is, it is assumed that the contribution of thermal kicks to the particle average to zero and the drag is the same as in a deterministic fluid. As we explain in this work, this is not necessarily so because of the nonlinear coupling between the moving particle and the fluctuating fluid.

For a spherical particle of radius $a$ suspended in a threedimensional fluid with shear viscosity $\eta$, the SE formula takes

\footnotetext{
a)Electronic mail: donev@courant.nyu.edu
}

the form

$$
\chi=\frac{k_{B} T}{\alpha \eta a},
$$

where $\alpha$ is a coefficient that depends on the boundary conditions applicable at the surface of the sphere, equal to $6 \pi$ for a stick surface and $4 \pi$ for a slip surface. ${ }^{1}$ In fact, the precise definitions of $a$ and $\alpha$ are ambiguous except in certain limiting cases, ${ }^{2,3}$ and it perhaps best to think of the product $\alpha a$ $=6 \pi R_{H}$ as a measure of the effective (stick) hydrodynamic radius of the particle $R_{H}$. It is not difficult to extend the SE relation to account for rotational diffusion and thus generalize it to more complicated rigid particle shapes. There is ample evidence and many theoretical calculations ${ }^{4,5}$ that demonstrate that (1) is asymptotically exact for a rigid sphere that is much larger and much more massive than the solvent molecules. Remarkably, the SE formula (1) is consistent with experimental and numerical measurements even for particles that are comparable in size to the solvent molecules (including a tagged fluid particle), with values of the hydrodynamic radius that are comparable to the actual physical size of the particle. Many deviations from this relation have been observed in particle simulations, ${ }^{3}$ however, it is virtually impossible to precisely attribute the cause of the mismatch because of the large number of violations of the assumptions that underlie (1). For example, traditional Navier-Stokes hydrodynamics may break down at the scales of the suspended particle, ${ }^{6}$ or the 
appropriate boundary conditions may be different from the traditional no-slip condition. ${ }^{2}$

Even if one assumes traditional hydrodynamics applies, there are additional assumptions that enter the SE relation. One of the most important, yet often overlooked, assumptions, is that the Schmidt number is very large, $\mathrm{Sc}=v / \chi$ $\gg 1$, where $v=\eta / \rho$ is the kinematic viscosity of the fluid. Physically, this means that momentum diffuses much faster than does the particle and the particle motion is viscousdominated. In particular, at large Schmidt numbers the fluid velocity quickly relaxes to the solution of the steady Stokes equation as the particle barely moves. ${ }^{7}$ This assumption can safely be made for realistic liquids. The Schmidt number for molecules in simple liquids is of the order of $10^{2}-10^{3}$, and for macromolecular or colloidal solutions it is at least an order of magnitude larger. However, there are several numerical methods used for simulating the diffusive motion of particles in flow for which this assumption cannot be safely made. This is particularly true for particle methods for hydrodynamics such as smooth particle hydrodynamics (SPH) ${ }^{8}$ smoothed dissipative particle dynamics (SDPD), ${ }^{9}$ stochastic hard-sphere dynamics (SHSD) ${ }^{10}$ and stochastic rotation dynamics (SRD) (also called multiparticle collision dynamics $\left.(\mathrm{MPCD})^{11}\right) \cdot{ }^{12,13}$ Achieving large Schmidt number in these methods requires a prohibitively small time step (collision frequency) and in many typical simulations Sc is less than 10. In several hydrodynamic (non-particle) methods such as the lattice Boltzmann (LB) method, ${ }^{14}$ the stochastic immersed boundary method, ${ }^{15,16}$ or the inertial coupling method used here, ${ }^{17} \mathrm{Sc}$ can be varied over a much broader range with comparative ease, however, Sc is still limited to moderate values (e.g., Sc $\sim 25-50$ ) by computational efficiency considerations due to the large separation of time scales between the viscous and diffusive dynamics. Practical heuristics have been developed empirically, ${ }^{18,19}$ with findings generally consistent with the detailed investigation we perform in this work.

In this work, we study the deviations of the diffusion coefficient of a tracer particle immersed in an incompressible Newtonian fluid from the Stokes-Einstein prediction at small and moderate Schmidt numbers. We utilize a recently developed incompressible inertial coupling method ${ }^{17}$ method for coupling minimally resolved particles with an incompressible fluctuating fluid solver. This allows us to eliminate compressibility yet consistently include both fluid and particle inertia, as well as the thermal fluctuations responsible for the diffusive Brownian motion. By changing the fluid viscosity we are able to control the Schmidt number and thus study whether the SE formula applies when the particle diffuses comparably fast to momentum. Furthermore, we can trivially change the dimensionality and study both two and three dimensional systems. There has been some confusion in the literature about the applicability of hydrodynamics to two dimensional systems, and statements to the effect that the SE relation does not apply in two dimensions have been made. ${ }^{20}$ As we will demonstrate, these misperceptions arise because finite-size effects diverge in two dimensions, ${ }^{21}$ and not because (fluctuating) hydrodynamics fails in two dimensions. In particular, we will demonstrate that in finite two dimensional systems the SE relation holds for $\mathrm{Sc} \gg 1$.
In Sec. II we describe the formulation of the minimally resolved model we use to describe the coupled particle-fluid system. We first discuss the emergence of the Stokes-Einstein result in the limit of infinite Schmidt number, and then, in Sec. II B, we discuss finite Schmidt number corrections. In Sec. III we present numerical results for the velocity autocorrelation function and long-time diffusion coefficient at finite Schmidt numbers, and then offer some conclusions in Sec. IV.

\section{BROWNIAN PARTICLE MODEL}

A detailed description of the fluid-particle equations that we employ, as well as a numerical algorithm to solve them, is given in Ref. 17. Here we briefly summarize the essential features, and then discuss in more detail the Brownian (overdamped) limit.

Let us consider a particle of physical mass $m$ and size (e.g., radius) $a$ immersed in a fluid with density $\rho$. The position of the particle is denoted with $\boldsymbol{q}(t)$ and its velocity with $\boldsymbol{u}=\dot{\boldsymbol{q}}$. The shape of the particle and its effective interaction with the fluid is captured through a smooth kernel function $\delta_{a}(\boldsymbol{r})$ that integrates to unity and whose support is localized in a region of size $a$. For example, one may choose any onedimensional "bell-shaped" curve $\delta_{a}(r)$ with half-width of or$\operatorname{der} a$, such as a normalized Gaussian with standard deviation $a$ or a symmetric function of compact support of half-width $a$. This kernel is used to mediate two crucial operations. First, it is used to transfer (spread) the force $\lambda$ exerted on the fluid by the particle to the fluid. Second, it is used to impose a minimally resolved form of the no-slip constraint stating that the velocity of the particle equals the local velocity of the fluid. We term this diffuse particle a blob for lack of better terminology (in polymer modeling the term bead is used for the same concept,${ }^{14}$ while blob is used to denote an effective soft particle that includes internal degrees of freedom). The physical volume of the blob $\Delta V$ is determined by the shape and width of the kernel function,

$$
\Delta V=\left[\int \delta_{a}^{2}(\boldsymbol{r}) d \boldsymbol{r}\right]^{-1} .
$$

The equations of motion in our inertial coupling method take the form, ${ }^{17}$

$$
\begin{gathered}
\rho\left(\partial_{t} \boldsymbol{v}+\boldsymbol{v} \cdot \nabla \boldsymbol{v}\right)=-\nabla \pi+\eta \nabla^{2} \boldsymbol{v}-\lambda \delta_{a}(\boldsymbol{q}-\boldsymbol{r}) \\
+\nabla \cdot\left[\left(k_{B} T \eta\right)^{\frac{1}{2}}\left(\mathcal{W}+\mathcal{W}^{T}\right)\right], \\
\boldsymbol{\nabla} \cdot \boldsymbol{v}=0, \\
m_{e} \dot{\boldsymbol{u}}=\boldsymbol{F}(\boldsymbol{q})+\lambda, \\
\boldsymbol{u}=\int \delta_{a}(\boldsymbol{q}-\boldsymbol{r}) \boldsymbol{v}(\boldsymbol{r}, t) d \boldsymbol{r}
\end{gathered}
$$

where $\rho$ is the constant fluid density, $\boldsymbol{v}(\boldsymbol{r}, t)$ is the incompressible fluid velocity, $\pi(\boldsymbol{r}, t)$ is the hydrodynamic pressure, $m_{e}$ is the excess mass of the particle relative to the fluid, and $\boldsymbol{F}(\boldsymbol{q})$ is the external force applied to the particle. 
Here the stochastic momentum flux is modeled using a whitenoise random Gaussian tensor field $\mathcal{W}(\boldsymbol{r}, t)$, that is, a tensor field whose components are independent (space-time) white noise processes. ${ }^{22}$ In this instantaneous frictionless coupling the total fluid-particle force $\lambda$ is a Lagrange multiplier that enforces the no-slip constraint (6). In this work we will exclusively consider periodic systems, i.e., consider diffusion of the Brownian particle on a torus, in either two or three dimensions. The equations used here only account for the hydrodynamic contribution to the diffusion coefficient, and not any additional molecular (kinetic) contributions. ${ }^{23}$ One can include a random slip to account for unresolved molecular effects, as described in the Appendix of Ref. 17, in which case the additional "bare" diffusion coefficient would simply be added to the hydrodynamic contribution studied here. ${ }^{24}$ In principle the equations employed here can be obtained by coarse-graining the complete microscopic dynamics with the momentum density field and the position of the particle as the relevant (slow) degrees of freedom. ${ }^{25}$

Let us now assume that the nonlinear inertial effects in the momentum equation can be neglected (this is easy to check in our method by simply omitting the advective momentum flux term in the implementation), and that the immersed particle is neutrally buoyant, $m_{e}=0$. In this work we will not carefully study the effects of the particle excess mass $m_{e}$, however, the results presented in Sec. III suggest that $m_{e}$ does not affect the long-time diffusive motion. This suggests that the dominant contribution to the effect we study here comes from the transient inertia of the fluid (i.e., the term $\rho \partial_{t} v$ in the momentum equation). Under these assumptions, we get the simpler equations of motion for the fluid-particle system,

$$
\begin{aligned}
\rho \partial_{t} \boldsymbol{v}+\nabla \pi & =\eta \nabla^{2} \boldsymbol{v}+\nabla \cdot\left[\left(k_{B} T \eta\right)^{\frac{1}{2}}\left(\mathcal{W}+\mathcal{W}^{T}\right)\right] \\
& +\boldsymbol{F}(\boldsymbol{q}) \delta_{a}(\boldsymbol{q}-\boldsymbol{r}) \\
\boldsymbol{u}=\frac{d \boldsymbol{q}}{d t} & =\int \delta_{a}(\boldsymbol{q}-\boldsymbol{r}) \boldsymbol{v}(\boldsymbol{r}, t) d \boldsymbol{r}
\end{aligned}
$$

which are much easier to analyze. Note, however, that even though the fluid equation is linearized, the no-slip constraint is a nonlinear constraint because of the presence of the particle position in the argument of the kernel. These semi-linear equations are also the equations used in the stochastic immersed boundary method ${ }^{15}$ and the closely related stochastic Eulerian-Lagrangian method. ${ }^{16}$ We note however that in our numerical calculations we employ the full equations (3)-(6), and only use the linearized equations for theoretical analysis. The numerical method used to solve the equations relies on a finite-volume staggered discretization of the fluid equation, ${ }^{26}$ and on the immersed boundary method ${ }^{27}$ for handling the fluid-particle interaction. ${ }^{17}$

The mobility of a blob is easy to evaluate in the deterministic setting (the stochastic setting will be discussed later on). Consider applying a constant force $\boldsymbol{F}$ on the blob and waiting for the velocity of the particle to reach a steady value. In the steady state the fluid velocity solves the steady Stokes equation and can be obtained explicitly,

$$
\boldsymbol{v}(\boldsymbol{r})=\eta^{-1} \int \boldsymbol{G}\left(\boldsymbol{r}, \boldsymbol{r}^{\prime}\right) \boldsymbol{F}(\boldsymbol{q}) \delta_{a}\left(\boldsymbol{q}-\boldsymbol{r}^{\prime}\right) d \boldsymbol{r}^{\prime},
$$

where $\boldsymbol{G}$ is the Green's function (Oseen tensor) for the steady Stokes equation ${ }^{49}$ with unit viscosity $\left(\nabla \pi=\nabla^{2} v+f\right.$ subject to $\nabla \cdot \boldsymbol{v}=0$ and appropriate boundary conditions). Note that for periodic boundaries the integral of $f$ over the unit periodic cell of volume $V$ must vanish; we therefore take the definition of $\boldsymbol{G}$ to include subtracting the total applied force $\boldsymbol{F}$ as a uniform force density $-V^{-1} \boldsymbol{F}$ on the right hand side of the momentum equation.

The velocity of the blob $\boldsymbol{u}=\boldsymbol{\mu} \boldsymbol{F}$ is determined from the no-slip constraint, giving the mobility tensor (see also Ref. 28)

$$
\boldsymbol{\mu}=\eta^{-1} \int \delta_{a}(\boldsymbol{q}-\boldsymbol{r}) \boldsymbol{G}\left(\boldsymbol{r}, \boldsymbol{r}^{\prime}\right) \delta_{a}\left(\boldsymbol{q}-\boldsymbol{r}^{\prime}\right) d \boldsymbol{r} d \boldsymbol{r}^{\prime}
$$

For isotropic systems the mobility tensor is a multiple of the identity tensor, $\boldsymbol{\mu}=\mu \boldsymbol{I}$. In three dimensions, the scalar mobility $\mu=(\alpha \eta a)^{-1}$ can be taken to define an effective hydrodynamic radius of the blob $R_{H} \sim a$ via the relation for a no-slip rigid sphere, $\mu \eta=\left(6 \pi R_{H}\right)^{-1}$. There are well-known finite-size corrections to the mobility for periodic systems with a unit cell of volume $L^{d}$ that scale like $L^{-1}$ in three dimensions. ${ }^{14,29,30}$ The hydrodynamic radius of the blobs that we employ in our numerical implementation has been measured computationally in Refs. 17 and 30. The spatial discretization of the fluid/particle equations leads to a small violation of translational invariance and isotropy and the mobility tensor is not exactly constant or diagonal but rather depends on the precise location of the blob relative to the underlying grid used to solve the fluid equation. ${ }^{17}$ These discretization artifacts are small, of the order of a couple percent for the three-point Peskin kernel, ${ }^{31}$ and a fraction of a percent for the four-point Peskin kernel. ${ }^{27}$ It is also possible to construct discrete kernel functions with even better translational invariance at the cost of increasing the support of the kernels and thus the computational cost of the algorithm.

In two dimensions, the mobility of a blob diverges logarithmically with system size, consistent with the Stokes paradox for flow past a cylinder in an unbounded domain. For periodic system with a square unit cell with area $L^{2}$, the logarithmic divergence of the Green's function for two-dimensional Stokes flow gives ${ }^{50}$

$$
\mu=(4 \pi \eta)^{-1} \ln \frac{L}{\alpha a},
$$

where the coefficient $\alpha$ depends on the shape of the kernel $\delta_{a} \cdot{ }^{21}$ This is analogous to the formula for the mobility of a periodic array of no-slip rigid cylinders of radius $R_{H}$, $\mu=(4 \pi \eta)^{-1} \ln \left[L /\left(3.708 R_{H}\right)\right],{ }^{29}$ and can be used to define an effective hydrodynamic radius for a two-dimensional blob. It is important to note that while the mobility diverges for an infinite system, for any finite system the mobility is finite and well-defined even in two dimensions. There is, in fact, no fundamental difference between two and three dimensions; it is simply the slower decay of the Green's function in two dimensions that changes the essential physics. 


\section{A. Brownian dynamics limit}

The short-time motion of particles immersed in the fluid is known to be very strongly affected by momentum diffusion and by inertial effects. ${ }^{32}$ Since our method includes inertial effects, it is able to produce both the correct short-time and long time features of the Brownian motion of a particle, where "short" refers to time scales after sound waves have decayed. This observation was made for the stochastic immersed boundary method in Refs. 7 and 28, and in Ref. 17 we demonstrated that our inertial coupling method also correctly captures the known physical effects of particle and fluid inertia on the velocity autocorrelation function (VACF)

$$
\boldsymbol{C}(t)=\langle\boldsymbol{u}(t) \otimes \boldsymbol{u}(0)\rangle,
$$

including the effect of particle excess mass on the short time behavior of the VACF, as well as the long time power-law tail of the VACF. Here we focus on the long-time diffusive motion of the particles, where "long" means that the motion is observed at a time scale $\tau$ over which momentum has diffused throughout the domain and the VACF has decayed to zero, $\tau>t_{L}=L^{2} / \nu$, where $v=\eta / \rho$ is the kinematic viscosity.

In the long-time limit, the motion of a single free particle immersed in a fluid looks like simple Brownian motion with a diffusion coefficient that can be defined from the long-time mean square displacement of the particle. More generally, one defines a time-dependent diffusion tensor, either using the mean-square displacement of the particle,

$$
\chi_{\mathrm{MSD}}(t)=\left\langle\frac{[\boldsymbol{q}(t)-\boldsymbol{q}(0)] \otimes[\boldsymbol{q}(t)-\boldsymbol{q}(0)]}{2 t}\right\rangle,
$$

or the integral of the VACF,

$$
\begin{aligned}
\chi_{\mathrm{VACF}}(t) & =\int_{0}^{t} \boldsymbol{C}\left(t^{\prime}\right) d t^{\prime} \\
& =\frac{d}{d t}\left\langle\frac{[\boldsymbol{q}(t)-\boldsymbol{q}(0)] \otimes[\boldsymbol{q}(t)-\boldsymbol{q}(0)]}{2}\right\rangle .
\end{aligned}
$$

The long-time diffusion tensor is then the asymptotic value

$$
\chi=\lim _{t \rightarrow \infty} \chi_{\mathrm{MSD}}(t)=\lim _{t \rightarrow \infty} \chi_{\mathrm{VACF}}(t) .
$$

While we will not demonstrate this here, it can be shown that, for very large Schmidt numbers, neither sound effects (compressibility) nor inertial effects affect the diffusion coefficient of a single particle. ${ }^{32}$ For a single particle, $\chi$ can be obtained from the mobility tensor $\boldsymbol{\mu}$ via the Einstein relation $\chi=\left(k_{B} T\right) \boldsymbol{\mu}$. It is important to note that the diffusive motion of the particle is entirely determined by its coupling to the fluid and is not an input parameter to our method. This reflects the physical relationship between fluid velocity fluctuations (viscosity and temperature) and diffusion coefficient, as encoded in the Stokes-Einstein relation. ${ }^{24}$

The Stokes-Einstein relation can formally be obtained starting from (7) by taking the overdamped limit $\nu=\eta / \rho \rightarrow$ $\infty$, which can be achieved by either assuming strong viscous friction $\eta \rightarrow \infty$, or no fluid inertia, $\rho \rightarrow 0$. In this limit, momentum diffuses much faster than does the immersed particles, as measured by the Schmidt number

$$
\mathrm{Sc}=\frac{v}{\chi} \gg 1
$$

The overdamped limit is the formal limit $\mathrm{Sc} \rightarrow \infty$, in which the fluid velocity becomes a fast degree of freedom that can be eliminated adiabatically, while the particle position becomes a slow degree of freedom. ${ }^{16,24,25}$ We emphasize that the StokesEinstein relation in a periodic domain of length $L \gg R_{H}$,

$$
\chi \rightarrow \chi_{\mathrm{SE}}=\left(k_{B} T\right) \begin{cases}(4 \pi \eta)^{-1} \ln \left[L /\left(3.708 R_{H}\right)\right] & \text { in two dimensions } \\ \left(6 \pi \eta R_{H}\right)^{-1} & \text { in three dimensions }\end{cases}
$$

can only be justified in the limit $\mathrm{Sc} \rightarrow \infty$.

For a collection of neutrally buoyant blobs, the multidimensional symmetric positive semi-definite mobility tensor $\boldsymbol{M}(\boldsymbol{Q})=\left\{\boldsymbol{\mu}_{i j}\right\}$ (which is a block matrix with blocks of size $d^{2}$, where $d$ is the dimensionality) depends on the positions of all particles $\boldsymbol{Q}=\left\{\boldsymbol{q}_{i}\right\}$. The diagonal block $\boldsymbol{\mu}_{i i}$ corresponds to the single-particle mobility in the absence of other particles, while the block corresponding to particle pair $i$ and $j$ is the inter-particle mobility $\boldsymbol{\mu}_{i j}$. For blobs this is given by a generalization of (8), ${ }^{28}$

$$
\boldsymbol{\mu}_{i j}=\boldsymbol{\mu}_{j i}=\eta^{-1} \int \delta_{a}\left(\boldsymbol{q}_{i}-\boldsymbol{r}\right) \boldsymbol{G}\left(\boldsymbol{r}, \boldsymbol{r}^{\prime}\right) \delta_{a}\left(\boldsymbol{q}_{j}-\boldsymbol{r}^{\prime}\right) d \boldsymbol{r} d \boldsymbol{r}^{\prime}
$$

This pairwise hydrodynamic interaction between two blobs was studied numerically for blobs in Ref. 17, and was shown to be closely related to the well-known Rotne-
Prager-Yamakawa (RPY) tensor used in Brownian dynamics simulations. ${ }^{18,33,34}$ In the overdamped limit, the collective diffusion of a collection of blobs can be described by the equations of Brownian dynamics, ${ }^{16,24}$

$$
\frac{d \boldsymbol{Q}}{d t}=\boldsymbol{M \mathcal { F }}+\sqrt{2 k_{B} T} \boldsymbol{M}^{\frac{1}{2}} \widetilde{\mathcal{W}}+k_{B} T\left(\frac{\partial}{\partial \boldsymbol{Q}} \cdot \boldsymbol{M}\right),
$$

where $\widetilde{\mathcal{W}}$ is a collection of independent white-noise processes and the noise is to be interpreted in the Ito sense, and $\mathcal{F}(\boldsymbol{Q})=\left\{\boldsymbol{F}_{i}\right\}$ are the forces applied on the particles. In this work we focus on the Brownian motion of a single particle, however, the effect of Schmidt number that we study here exists in multi-particle systems as well.

It is important to point out that at times longer than the time $t_{L}=L^{2} / \nu$ it takes for momentum to traverse the system length $L$, the VACF starts decaying exponentially ${ }^{7}$ and 
$\chi_{\mathrm{VACF}}\left(t>t_{L}\right) \approx \chi$. Therefore, at times $t>t_{L}$ the diffusive motion of an isolated particle looks like simple Brownian motion and it becomes meaningful to use the long-time diffusion coefficient. Note however that the Brownian motions of multiple particles are not independent because of the correlations induced by the long-ranged (decaying as $\ln (r)$ in two dimensions and $r^{-1}$ in three dimensions) hydrodynamic interactions. The diffusion of a large collection of particles is therefore subtly but crucially different from that of a collection of independent Brownian walkers with diffusion coefficient $\chi .^{24}$

\section{B. Finite schmidt numbers}

For finite Schmidt numbers, $\mathrm{Sc}=O(1)$, theoretical analysis is significantly complicated by the fact that the particle moves while the velocity relaxes through viscous dissipation. We are not aware of any rigorous results regarding the diffusive motion of even a single particle, yet alone collective diffusion. The main theoretical approach in the literature is mode-mode coupling theories, ${ }^{35}$ which are essentially a perturbative series in the strength of the thermal fluctuations. One key prediction of these theories is that the momentum diffusion coefficient $v=\eta / \rho$ should be augmented by the particle diffusion coefficient $\chi$ since the particle diffuses while the momentum diffuses. At the viscous time scale, $t>t_{v}=R_{H}^{2} / v$, conservation of momentum (hydrodynamics) in the fluid introduces a memory in the motion of the particle and the VACF $C(t)=d^{-1}\langle\boldsymbol{u}(t) \cdot \boldsymbol{u}(0)\rangle$ decays algebraically rather than exponentially. The standard theory for the tail of the VACF (long-time behavior) ${ }^{32}$ implicitly assumes that Sc $\gg 1$, and leads to the conclusion that for an isolated particle in an infinite fluid asymptotically $C(t) \approx\left(t / t_{v}\right)^{-d / 2}$ $\sim(v t)^{-d / 2}$. Self-consistent mode coupling theory predicts that at finite Schmidt numbers $C(t) \sim[(\chi+v) t]^{-d / 2}$. 5,36 This was confirmed to hold for blob particles numerically in Ref. 17 for Sc $\gtrsim 2$, with the caveat that $\chi$ was approximated by $\chi_{\mathrm{SE}}$, the prediction of the Stokes-Einstein relation.

It is not difficult to see that predictions of mode-mode coupling theories have to be approximate in nature since the diffusion coefficient of the particle, which is the result of the fluid-particle coupling, is used in the theory as an input to predict the corrections to the overdamped limit. Self-consistent mode-mode coupling theories are usually heuristic and thus also approximate. Based on the scaling of the tail of the VACF with Schmidt number, and the fact that the diffusion coefficient is given by the integral of the VACF, one may conjecture that, to leading order, the effect of finite Schmidt number can be captured by the modified Stokes-Einstein formula

$$
\chi(\nu+\chi)=\chi_{\mathrm{SE}} v .
$$

This relation was proposed as a self-consistent equation for $\chi$ in Refs. 37 and 38. It is important to note, however, that both the short time and the long time part of the VACF contribute to the diffusion coefficient. Numerical results in Ref. 17 indicate that the short-time VACF does not scale in the same way as the long-time tail, leading us to question the prediction (12). In particular, at very short times no rescaling is required to overlap the VACFs, and therefore, in principle, to fully overlap the VACFs over both short and long times, one would need to use an Sc-dependent non-uniform rescaling of time axes. One therefore expects the integral of the VACF to be somewhere in-between the Stokes-Einstein relation (corresponding to the short-time scaling) and (12) (corresponding to the long-time scaling).

We are not aware of any detailed theory that successfully predicts the form of the corrections to the simple StokesEinstein prediction (1) for moderate values of Sc (see Ref. 5 for one attempt). In Ref. 21 a heuristic self-consistent theory of diffusion is proposed. The starting point is renormalization theory for the effective diffusion coefficient in the advectiondiffusion equation for the concentration of a large number of passive tracers. ${ }^{51}$ The self-consistent theory suggests that in both two and three dimensions (cf. Eqs. (29) and (30) in Ref. 21 in the case of no bare diffusion)

$$
\chi=v\left[1+2 \frac{\chi_{\mathrm{SE}}}{v}\right]^{1 / 2}-v=v\left[\left(1+\frac{2}{\mathrm{Sc}^{\mathrm{SE}}}\right)^{\frac{1}{2}}-1\right],
$$

where we have defined a predicted Schmidt number $\mathrm{Sc}^{\mathrm{SE}}=v / \chi_{\mathrm{SE}}$ (which is input to our simulations rather than output as is Sc). This prediction for the self-diffusion coefficient is the solution to the self-consistent equation

$$
\chi\left(v+\frac{\chi}{2}\right)=\chi_{\mathrm{SE}} v,
$$

which differs from (12) in the prefactor of 1/2. In Sec. III, we will compare the two predictions (12) and (14) for the leadingorder correction to Stokes-Einstein's relation with numerical results. Note that we only expect this type of relation to predict the leading-order corrections (proportional to $\mathrm{Sc}^{-1}$ ) to the Stokes-Einstein relation and not the complete dependence on Sc. At very small Schmidt numbers there would likely be important contributions from higher order (e.g., proportional to $\chi^{2} / \nu$ ) correction terms inside the parenthesis in (14).

Mode-mode and renormalization theories, on which both (12) and (14) are based, are perturbative theories typically truncated at terms quadratic in the strength of the fluctuations. In the context of infinite (bulk) systems, a systematic perturbative theory that accounts for corrections of order higher than quadratic in the fluctuations has been discussed in Refs. 38 and 39. In three dimensions, the conclusion of such studies has been that the higher order terms do not make a dramatic contribution. This can be attributed to the fact that large-scale modes of the fluctuating velocity make a negligible contribution to the diffusive dynamics (this is directly related to the fact that the $t^{-3 / 2}$ tail of the VACF is integrable, see Ref. 21 for additional discussion).

In two dimensions, however, the logarithmic divergence of $\chi_{\mathrm{SE}}$ with system size is a sign of the growing contribution of large-scale modes. In fact, the Schmidt number will become arbitrarily small for sufficiently large systems (keeping viscosity fixed) and the Stokes-Einstein relation will be strongly violated. In this case, however, the infinite-time diffusion coefficient $\chi$ does not describe the diffusive dynamics even at macroscopic length (e.g., system size $L$ ) and time scales $\left(L^{2} / \chi\right)$. Specifically, the slowly decaying $t^{-1}$ tail of the VACF means that the diffusive motion has correlations over 
macroscopic times and does not resemble simple Brownian motion at relevant scales. Instead, one needs to consider the time-dependent diffusion coefficients $\chi_{\mathrm{MSD}}(t)$ or $\chi_{\mathrm{VACF}}(t)$. Several calculations ${ }^{39-41}$ and numerical simulations ${ }^{42,43}$ suggest that including higher order terms changes the decay of the tail of the VACF in two dimensions. Specifically, it has been predicted that the self-consistent power-law decay for the VACF is faster than $t^{-1}, C(t) \sim(t \sqrt{\ln t})^{-1}$. Hydrodynamics-based methods such as the method we use here (see also Ref. 42 for a study using a lattice gas model) are better for studying this very long time decay of the VACF than are particle methods, since larger systems and longer time scales become computationally feasible. Nevertheless, because of the slow logarithmic growth, extremely large systems are required to see any effects of higher order corrections. To see this, let us assume Stokes-Einstein's formula holds and estimate the system size when

$$
\mathrm{Sc} \approx \mathrm{Sc}^{\mathrm{SE}}=\frac{v}{\chi_{\mathrm{SE}}}=\frac{4 \pi \rho v^{2}}{k_{B} T \ln \left[L /\left(4 R_{H}\right)\right]}<1 .
$$

For a hard-disk fluid, we can estimate the viscosity $\eta$ using simple Enskog kinetic theory, ${ }^{44}$ which has been found to be quite accurate even at high densities. ${ }^{45}$ For a hard-disk fluid at packing density (fraction) $\phi=(\rho / m)\left(\pi \sigma^{2} / 4\right)=0.6$ (which is close to the liquid-solid transition), Enskog theory predicts

$$
\eta \approx 2 \frac{\sqrt{m k_{B} T}}{\sigma}
$$

where $\sigma \approx 2 R_{H}$ denotes the particle diameter. This gives that the system size required to get Schmidt number close to unity,

$$
\mathrm{Sc}^{\mathrm{SE}} \approx \frac{65}{\ln \left[L /\left(4 R_{H}\right)\right]}<1 \Rightarrow L>1.5 \cdot 10^{29} R_{H}
$$

Reaching such a staggering system size is not feasible with any numerical method, and therefore in this work we focus on the more practically relevant case of finite system size at moderately large Schmidt numbers.

In addition to the nonlinearity coming from the fact that at moderate Schmidt numbers the particle moves while momentum diffuses, ${ }^{52}$ additional nonlinearities arise because of the presence of the advective term $\rho \boldsymbol{v} \cdot \nabla \boldsymbol{v}$ in the momentum equation. If we define a "thermal" Reynolds number based on the equilibrium magnitude of the particle thermal velocity fluctuations ${ }^{17}$

$$
\left\langle u^{2}\right\rangle^{\frac{1}{2}} \approx \sqrt{\frac{k_{B} T}{\rho R_{H}^{d}}}
$$

in three dimensions we can estimate

$$
\mathrm{Re}=\frac{\left\langle u^{2}\right\rangle^{\frac{1}{2}} R_{H}}{v} \approx \sqrt{\frac{k_{B} T}{v \eta R_{H}}} \sim \sqrt{\frac{\chi_{\mathrm{SE}}}{v}}=\left(\mathrm{Sc}^{\mathrm{SE}}\right)^{-\frac{1}{2}} .
$$

A similar result applies in two dimensions as well, except for an additional factor of $\ln ^{\frac{1}{2}}\left[L /\left(4 R_{H}\right)\right]$. This shows that the thermal Reynolds number becomes $O(1)$ when $\mathrm{Sc}^{\mathrm{SE}}$ $\sim 1$, and in principle nonlinear effects arising from advection could arise. By contrast, at large Schmidt numbers the thermal Reynolds number is small and advective nonlinearities are expected to be negligible. While in realistic fluids, including particle simulations, advective nonlinearities are always present, in our numerical method we can simply turn off the term $\rho \boldsymbol{v} \cdot \nabla \boldsymbol{v}$ to assess its importance. Numerical experiments indicate that nonlinear inertial effects play a minimal role, not noticeable within statistical accuracy for reasonable Schmidt numbers. Therefore, it appears appropriate to simply linearize the velocity equation, as is often done in the literature with somewhat hand-waving justifications. Similarly, we find that the excess inertia of the particles does not affect the long-time diffusion coefficient, consistent with traditional derivations based on linearized theory. ${ }^{32}$ We do not attempt to provide rigorous justification for these observations in this work.

\section{RESULTS}

In this section we present numerical results obtained using the computational algorithm for solving (3)-(6) developed in Ref. 17. In Ref. 17 some of us presented results concerning the short-time behavior of the VACF $C(t)$ of a single blob particle in a three-dimensional periodic domain of length $L$, including a comparison to the case of a blob suspended in a compressible fluid. Excellent agreement with theoretical predictions for the variance of velocity (i.e., $C(t=0)$ ) was obtained, and the $t^{-3 / 2}$ power-law behavior at long times $L^{2} / v>t>t_{v}=R_{H}^{2} / v$ was confirmed. Here we briefly examine the VACF in two dimensions, and then focus on the self-diffusion of a blob in both two and three dimensions. Note that in Ref. 17 it is demonstrated that the method correctly reproduces the static structure of a suspension of many interacting blobs. Also note that the presence of multiple interacting blobs affects the dynamics in a nontrivial way, and changes both the short-time VACF (via multi-particle inertial effects) and also the self-diffusion coefficient. We do not address multi-particle suspensions in this work.

In the majority of the simulations we use the three-point discrete kernel function of Roma and Peskin ${ }^{30,31}$ to discretize the kernel $\delta_{a}$. By using the Peskin four-point kernel ${ }^{27}$ instead of the three-point discrete kernel function the translational invariance of the spatial discretization can be improved, however, at a potentially significant increase in computational cost, particularly in three dimensions. The effective hydrodynamic radius $R_{H}$ for a given discrete kernel function can be obtained easily from the deterministic mobility tensor $\mu_{\mathrm{det}}$, given by a discrete equivalent of (8). The deterministic mobility tensor can be obtained by turning off fluctuations, applying a unit force along each of the coordinate directions in turn, solving the spatially discretized steady Stokes equation, and then calculating the resulting velocity of the particle. After accounting for finite-size effects due to the finite length of the periodic box, in three dimensions we numerically estimate ${ }^{17,30}$ the effective hydrodynamic radius to be $R_{H}^{3 p t}=(0.91 \pm 0.01) h$ the three-point kernel, ${ }^{53}$ where $h$ is the grid spacing, and $R_{H}^{4 p t}=(1.255 \pm 0.005) h$ for the $4 \mathrm{pt}$ kernel. ${ }^{46}$ In two dimensions, the effective (rigid disk) hydrodynamic radii are estimated to be $R_{H}^{3 p t}=(0.72 \pm 0.01) h$ and $R_{H}^{4 p t}=(1.04 \pm 0.005) h$. Note that the spatial discretization we use is not perfectly translationally invariant and there is 
a small variation of $R_{H}$ (quoted above as an error bar) as the particle moves relative to the underlying fixed fluid grid. ${ }^{17,46}$

We use a relatively small grid of $32^{2}$ cells in two dimensions and $32^{3}$ cells in three dimensions in order to be able to perform sufficiently long runs even with the larger Schmidt numbers. In two dimensions we use a neutrally buoyant particle $\left(m_{e}=0\right)$, while in three dimensions we use a particle twice denser than the surrounding fluid $\left(m_{e}=\rho \Delta V\right)$, in order to confirm that the excess mass (density) does not (significantly) affect the conclusions of our investigations. The advantage of using neutrally buoyant blobs is that they are passive tracers (they do not affect the velocity equation), and therefore one can use multiple tracers in a single simulation in order to improve the statistical accuracy. This is useful in two dimensions, where the VACF has a slowly decaying tail and therefore long runs are required to study the longtime diffusive motion of the particle. In all cases we have confirmed that the time step size $\Delta t$ is sufficiently small by comparing with a simulation using a twice smaller time step size. A detailed discussion and numerical results concerning the accuracy of the scheme as a function of $\Delta t$ are given in Ref. 17. We varied the viscosity in order to change the Schmidt number, but as explained earlier, the Schmidt number is the only relevant dimensionless number so one can equivalently change the temperature. Since the actual Schmidt number is an output rather than an input to our calculation, we cannot calculate the Schmidt number a priori. Therefore, in this section we estimate the true Schmidt number with $\mathrm{Sc} \approx \mathrm{Sc}^{\mathrm{SE}}$ $=v / \chi_{\mathrm{SE}}$ for the purposes of captions and axes labels, which is a good approximation for moderate and large Schmidt numbers.

During each simulation a discrete trajectory of the particle $\{\boldsymbol{q}(0), \boldsymbol{q}(\Delta t), \boldsymbol{q}(2 \Delta t), \ldots\}$ is recorded. From this data, we calculate an apparent (discrete) velocity $\tilde{\boldsymbol{u}}_{k}$ $=(\boldsymbol{q}(k+1)-\boldsymbol{q}(k)) / \Delta t, k=1,2, \ldots$, which for finite $\Delta t$ is in general different from the velocity of the particle $\boldsymbol{u}(k \Delta t)$ calculated by the numerical scheme. We then obtain the discrete VACF $C_{k}=d^{-1}\left\langle\left(\tilde{\boldsymbol{u}}_{k^{\prime}}\right) \cdot\left(\tilde{\boldsymbol{u}}_{k^{\prime}+k}\right)\right\rangle$ efficiently using a fast Fourier transform of the apparent velocity. It is not hard to show that the time-dependent diffusion coefficients at the discrete time points in time can be obtained in linear time using

$$
\begin{gathered}
\chi_{\mathrm{MSD}}(k \Delta t)=\frac{\Delta t}{2} C_{0}+\Delta t\left[\sum_{i=1}^{k-1} C_{i}-\frac{1}{k} \sum_{i=1}^{k-1} i \cdot C_{i}\right] \\
\chi_{\mathrm{VACF}}(k \Delta t) \approx \frac{\Delta t}{2} C_{0}+\Delta t \sum_{i=1}^{k-1} C_{i} .
\end{gathered}
$$

\section{A. VACF}

In Fig. 1 we show the VACF $C(t)=d^{-1}\langle\boldsymbol{u}(t) \cdot \boldsymbol{u}(0)\rangle$ for a neutrally buoyant $\left(m_{e}=0\right)$ blob in two dimensions, for several Schmidt numbers. The theoretical variance of the velocity of a neutrally buoyant particle immersed in an incompressible two dimensional fluid gives $C(t=0)=(d-1) k_{B} T /(d m)$ $=k_{B} T /(2 \rho \Delta V)$, where $\Delta V$ is the volume of the blob given in Eq. (2). We see from the figure that the numerical curves

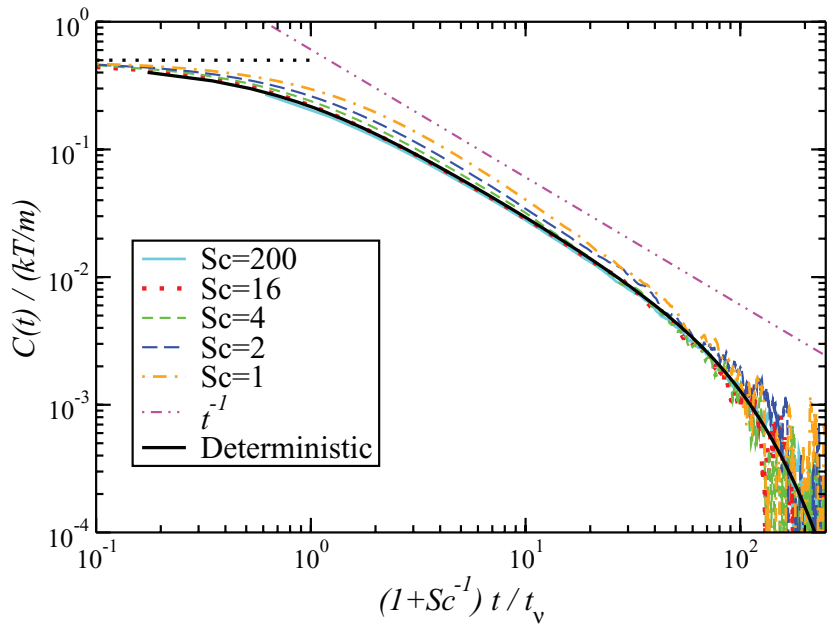

FIG. 1. Velocity autocorrelation function (VACF) of a single neutrally buoyant blob (three-point kernel) in two dimensions. A deterministic calculation, corresponding to the limit $\mathrm{Sc} \rightarrow \infty$, is also shown. The expected value at the origin is shown with a dotted line. The scaling of the time axes is chosen to collapse the different curves at long times. Note that the time $t_{L}=L^{2} / v$ $\approx 10^{3} t_{v}$ is off the scale of the time axis.

are in excellent agreement with this value, confirming that the numerical method correctly captures the thermal particle velocity fluctuations.

The standard theory for the tail of the VACF (long-time behavior $)^{32}$ implicitly assumes that $\mathrm{Sc} \gg 1$, and predicts that the long-time VACF has a power law decay $C(t) \sim(v t)^{-1}$. As discussed in Sec. II B, accounting for a finite Schmidt number leads to a predicted decay $C(t) \sim[(v+\chi) t]^{-1}$. This means that the tails of the VACFs for different $\mathrm{Sc}$ values can be collapsed on one master curve if we plot them as a function of $\left(1+\mathrm{Sc}^{-1}\right)\left(t / t_{v}\right)$. This was confirmed in Ref. 17 in three dimensions, and it is confirmed in two dimensions in Fig. 1. The rescaling is not perfect at shorter times, especially for small Schmidt numbers, however, we note that the collapse is significantly poorer if one plots the VACF as a function of just $t / t_{v}$.

Our numerical method becomes very inefficient as the Schmidt number becomes very large due to the large separation of scales between the momentum and particle diffusion. We will present modifications of the numerical method to handle the limit $\mathrm{Sc} \rightarrow \infty$ in future work. The VACF in the limit Sc $\rightarrow \infty$ can, however, easily be obtained by a deterministic calculation. We simply give the particle a small momentum kick $\Delta p=F \Delta t$ during the first time step, and then the deterministic algorithm is used to track the subsequent decay of the velocity $u(t)$, which gives the VACF in the limit after a suitable rescaling. In particular, the time-dependent diffusion coefficient in the limit $\mathrm{Sc} \rightarrow \infty$ is given by

$$
\lim _{\mathrm{Sc} \rightarrow \infty} \chi_{\mathrm{VACF}}(t)=\frac{k_{B} T}{\Delta p} \int_{0}^{t} u\left(t^{\prime}\right) d t^{\prime} .
$$

In Fig. 1 we show also the result of such a deterministic calculation. Due to lack of statistical noise, the deterministic VACF shows very clearly the transition from a power-law to an exponential tail at long times. It is also clearly seen that the VACF at large Schmidt numbers closely matches the 
deterministic one, as expected. Note that, in principle, the deterministic VACF can be calculated analytically using discrete Fourier Transform techniques. ${ }^{7}$

\section{B. Diffusion coefficient}

The long-time diffusion coefficient can be obtained either from the limiting value of $\chi_{\mathrm{MSD}}(t)$ or $\chi_{\mathrm{VACF}}(t)$. It is preferable to use the discrete integral of the (discrete) VACF rather than the mean square displacement because $\chi_{\mathrm{VACF}}$ converges faster to the asymptotic (long-time) value, ${ }^{54}$ and thus has smaller statistical error. Because the position of the particle is a more fundamental quantity (in particular, in the limit $\mathrm{Sc} \rightarrow \infty$ it becomes the only relevant variable), the mean square displacement has a more direct physical interpretation, and is directly obtained from the discrete particle trajectory. We therefore use $\chi_{\mathrm{MSD}}(t)$ to illustrate the time dependence of the diffusion coefficient, and use $\chi_{\mathrm{VACF}}(t)$ to obtain the long-time diffusion coefficient $\chi$.

In the left panel of Fig. 2 we show the time-dependent diffusion coefficient $\chi_{\mathrm{MSD}}(t)$ for a single particle in a three dimensional periodic domain at several Schmidt numbers, including the limit Sc $\rightarrow \infty$ as obtained from a deterministic simulation. We see that at short times we obtain collapse of all of the curves just by scaling the time axes by $t_{v}=R_{H}^{2} / \nu$, however, at long times the curves do not collapse. In particular, the diffusion coefficient is lower than the Stokes-Einstein prediction for the smaller Schmidt numbers. In order to obtain an estimate of the long-time diffusion coefficient that is essentially converged to the asymptotic value, while at the same time minimizing the statistical errors, we estimate $\chi \approx \chi_{\mathrm{VACF}}\left(L^{2} /(4 v)\right)$. In the right panel of Fig. 2 we show the estimated $\chi / \chi_{\mathrm{SE}}$ as a function of the approximate Schmidt number $\mathrm{Sc}^{\mathrm{SE}}$. The numerical data are compared to the two self-consistent theories, (12) and (14). We see good agreement of the numerical data with (14) to within the statistical accuracy.

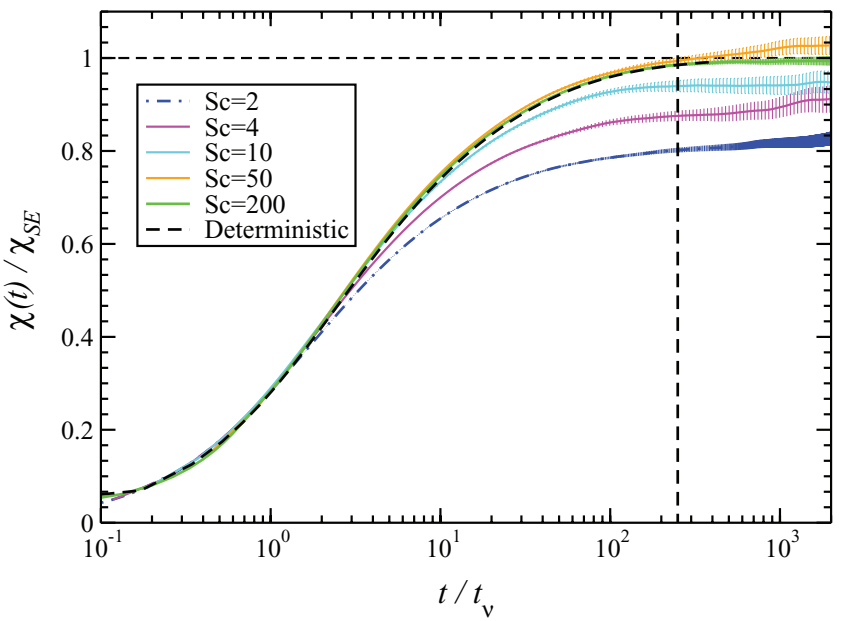

Our numerical results clearly demonstrate that the Stokes-Einstein relation does not apply at finite Schmidt numbers. On the other hand, the Einstein formula, which is a linear response relationship between the diffusion coefficient and the mobility, $\chi=\left(k_{B} T\right) \mu$, is quite general, and follows from straightforward statistical mechanics arguments. ${ }^{47}$ However, one must be careful here in defining the mobility $\mu$. Mobility should be defined as the coefficient of proportionality between a small applied force $F_{0}$ on the particle (with the opposite force applied uniformly to the fluid to prevent center-of-mass motion) and the average velocity of the particle,

$$
\mu=\lim _{F_{0} \rightarrow 0} \frac{\langle\boldsymbol{u}\rangle}{\boldsymbol{F}_{0}}
$$

in the presence of thermal fluctuations. Here the average is taken over time, or, equivalently, over a nonequilibrium but steady-state ensemble that is a weak perturbation of the equilibrium ensemble. Because of the nonlinearity of the fluidparticle equations, thermal fluctuations can affect average values. Therefore, we should not expect, in general, that the mobility measured at finite temperature is the same as the deterministic $\left(k_{B} T=0\right)$ mobility. Indeed, in the right panel of Fig. 2 we show the predictions of (17) with $\langle\boldsymbol{u}\rangle$ measured numerically under a small applied force (to ensure the system remains in the linear response regime). These predictions are in agreement with the direct measurements of the diffusion coefficient from the integral of the velocity autocorrelation function, as predicted by linear response theory. We therefore observe no violation of any known physical principles, so long as we recognize the fact that fluctuations cannot just be turned off. More precisely, at finite value of $k_{B} T$ the fluctuations do not necessarily average out, and can, in fact, affect the dynamics of macroscopic or deterministic observables.

In Fig. 3 we confirm that the same conclusions apply to finite two-dimensional systems. In order to verify that the results are not affected by the specific choice of the kernel function, here we also use the four-point Peskin kernel. ${ }^{27}$ The

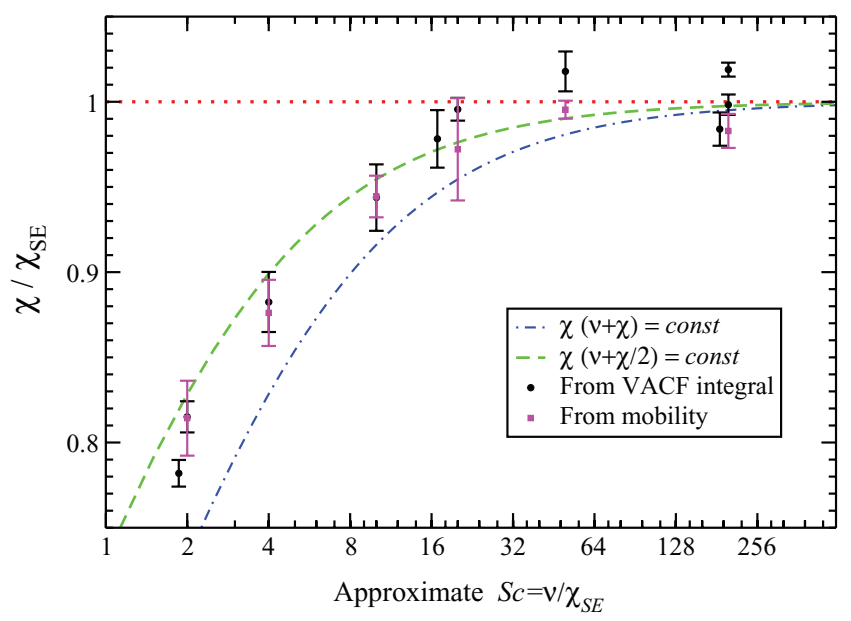

FIG. 2. (Left panel) The ratio of the time-dependent diffusion coefficient $\chi_{\mathrm{MSD}}(t)$ to the long-time diffusion coefficient estimate $\chi_{\mathrm{SE}}$ given by the StokesEinstein formula, for a three dimensional blob (three-point kernel). The statistical error estimates are shown as vertical bars, and the vertical line shows the time $\tau=L^{2} /(4 v)$ at which we estimate the long-time diffusion coefficient $\chi \approx \chi_{\mathrm{VACF}}(\tau)$. (Right panel) Comparison between the numerical estimate (symbols) for the long-time diffusion coefficient and the Stokes-Einstein prediction for several Schmidt numbers. The predictions of the self-consistent theories (12) and (14) are shown for comparison (dashed lines). The Einstein prediction $\chi=\left(k_{B} T\right) \mu$ based on direct measurements of the mobility (17) are shown for several Schmidt numbers. 

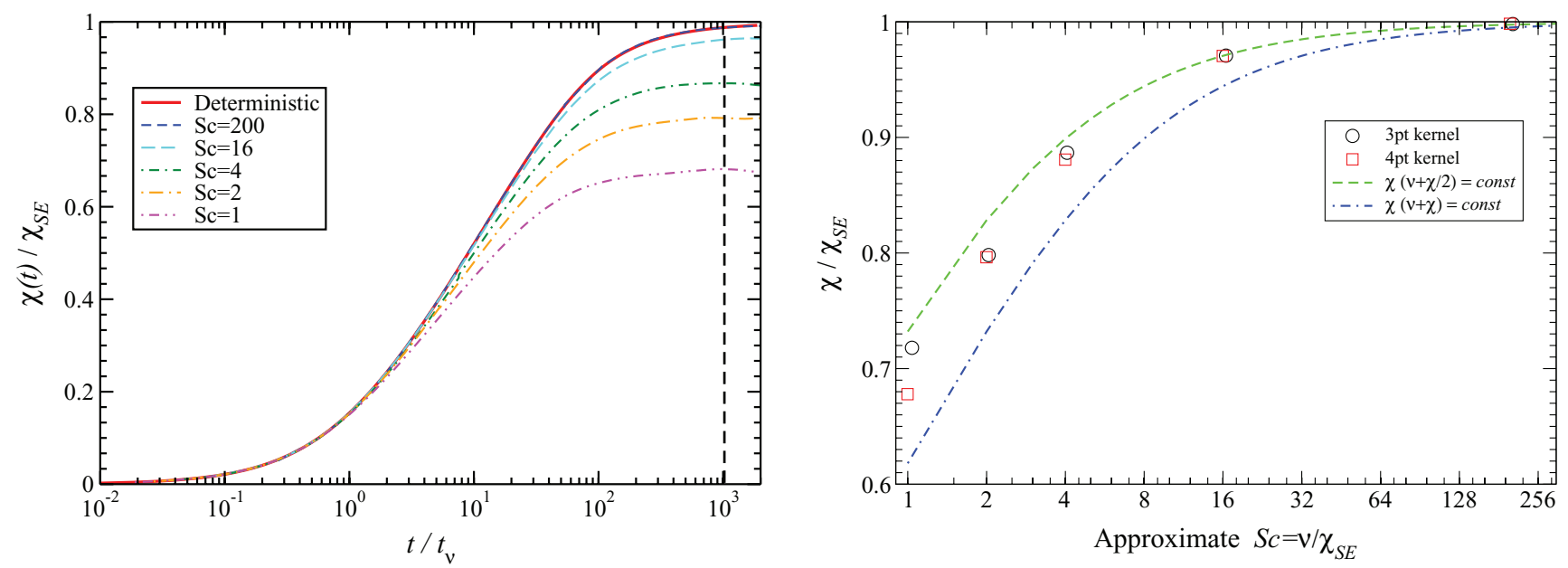

FIG. 3. The same panels as Fig. 2 but this time for a two dimensional blob. The four-point kernel was used for the left panel, and both the three- and four-point kernels are employed for the right panel.

results in the right panel of Fig. 3 show that dimensionless relations are independent of the kernel function. This leads us to believe that (14) is a rather general relation that correctly captures the leading-order corrections to the Stokes-Einstein relation at finite Schmidt numbers in both two and three dimensions.

\section{CONCLUSIONS}

We presented a simple theory for the effect of Schmidt number $\mathrm{Sc}=v / \chi$ on the long-time diffusion coefficient of a particle immersed in a fluid. The approximate selfconsistent calculation suggests that the long-time diffusion coefficient of the particle can be determined from the condition $\chi(v+\chi / 2)=\chi_{\mathrm{SE}} v$. Here $\chi_{\mathrm{SE}}$ is the prediction of the Stokes-Einstein relation, $\chi_{\mathrm{SE}}=k_{B} T \mu_{\text {det }}$, where $\mu_{\text {det }}$ is the deterministic mobility of the particle, that is, the linear response to an applied force in the absence of thermal fluctuations. In the limit of large Schmidt numbers the StokesEinstein relation is obeyed, as expected, but the correction can be substantial at small Schmidt numbers and is also measurable at moderate Schmidt numbers. By contrast, the Einstein relation $\chi=k_{B} T \mu$ is always obeyed, as long as the mobility is defined by the linear response in the presence of fluctuations. The discrepancy between $\mu$ and $\mu_{\text {det }}$ stems from the nonlinear fluid-particle coupling, which becomes important at finite Schmidt numbers.

Our numerical results are in good agreement with the self-consistent theory in both two and three dimensions. In two dimensions, for finite systems, Stokes-Einstein's relation holds, but the Stokes-Einstein diffusion coefficient diverges logarithmically with system size. At the same time, however, as the system size diverges the Schmidt number decreases and the deviations from Stokes-Einstein's prediction become stronger. The truly asymptotic behavior of a particle in an unbounded two-dimensional fluid (e.g., a tagged hard disk in a hard-disk fluid) remains an open question, albeit one of mostly academic interest since the Schmidt number is still rather large for system sizes feasible in present-day molecular dynamics.
While the above results elucidate some important fundamental questions about the physical importance of fluctuations, it should be observed that for realistic fluids (rather than coarse-grained fluid models ${ }^{11}$ ) small Sc implies that the size of the blob particle becomes comparable to the length scale at which hydrodynamics breaks down (molecular diameter for liquids, or mean free path for gases). The physical fidelity of the blob model and the fluctuating hydrodynamics equations ${ }^{11}$ used here should therefore be questioned at small Sc. In Ref. 3 the validity of the Stokes-Einstein relation is studied using molecular dynamics. In that study the smallest Schmidt number is of the order of 15 , and the corrections studied here are significant. Nevertheless, in the case of a true molecular fluid many other effects enter the picture as well, for example, the (in)validity of the no-slip condition with a fixed particle radius. Therefore, one should not try to explain the data in Ref. 3 using our model. In fact, the deviations from the SE prediction measured in Ref. 3 have a comparable magnitude but the opposite sign from the correction predicted here. This means that, when one takes into account that hydrodynamic theory should include the finite Schmidt-number correction studied here, the deviations between the MD simulation and hydrodynamic predictions are twice larger than reported in Ref. 3. A similar "cancellation-of-errors" that leads to a better agreement with hydrodynamic theory than truthfully present is discussed in Ref. 2 .

Despite its somewhat limited practical utility, our model allows us to separate hydrodynamic from molecular effects, and to test the predictions of self-consistent renormalization theory. ${ }^{21}$ More importantly, our results are quite relevant to the interpretation of results obtained using widely used particle methods for fluid dynamics of complex fluids, e.g., MD, (S)DPD, MPCD/SRD, SHSD, and even Lattice Boltzmann schemes. In some of these methods, as they are typically used, the Schmidt number can be of the order of unity, and in all of them it is rarely larger than 50 . This is to be contrasted with realistic liquids (e.g., water at room conditions) where the Schmidt number for tagged particles exceeds 100 and is often larger than 1000. In order to avoid the types of unphysical deviations from the Stokes-Einstein relation as we described 
here, one must use methods that take the limit Sc $\rightarrow \infty$ and use a fluctuating steady Stokes equation for the fluid (e.g., Brownian and Stokesian dynamics). In the future we will develop modifications of our method that achieves this limiting behavior. Note that increasing Sc is not possible in traditional particle methods or Lattice Boltzmann methods because they (by design) avoid the solution of Poisson equations for the pressure, as is necessary in the steady Stokes limit.

The primary cause of the correction to the SE prediction at finite $\mathrm{Sc}$ is the fact that the particle diffuses with effective diffusion coefficient $\chi$ while the momentum diffuses, rather than nonlinear inertial effects. This leads us to suspect that the effect studied here is independent of the details of the microscopic dynamics giving rise to the particle diffusion, and the same self-consistent formula should apply, for example, in Lattice Boltzmann methods with a frictional fluid-particle coupling. ${ }^{14}$ Note that in Stokes frictional coupling, and likely also in molecular (particle) simulations, there is an additional non-hydrodynamic or "bare" contribution to the diffusion coefficient $\chi_{0}=k_{B} T / \gamma$ coming from the dissipative friction coefficient $\gamma$ between the particle and the fluid. ${ }^{14,23}$ A way to estimate such bare friction in molecules, from the autocorrelation of molecular forces in restraint dynamics, has been proposed in Ref. 48 . The bare diffusion coefficient $\chi_{0}$ should be added to the (Schmidt-number dependent) hydrodynamic contribution $\chi_{H}=\chi-\chi_{0}$ studied in this work. In both two and three dimensions, the self-consistent theory proposed in Ref. 21 gives the prediction

$$
\chi=\chi_{0}+\left[1+\frac{\chi+\chi_{0}}{2 v}\right]^{-1} \chi_{\mathrm{SE}}
$$

which can be written as a self-consistent equation for $\chi_{H}$,

$$
\chi_{H}\left(v+\chi_{0}+\frac{\chi_{H}}{2}\right)=\chi_{\mathrm{SE}} v .
$$

It would be interesting to perform numerical simulations in the future to test this theory numerically.

\section{ACKNOWLEDGMENTS}

We thank Burkhard Dünweg and Anthony Ladd for informative discussions. A. Donev was supported in part by the Air Force Office of Scientific Research under Grant No. FA955012-1-0356. R. Delgado-Buscalioni and F. Balboa acknowledge funding from the Spanish Government FIS2010-22047$\mathrm{C} 05$ and from the Comunidad de Madrid MODELICO-CM (S2009/ESP-1691). Collaboration between A. Donev and R. Delgado-Buscalioni was fostered at the Kavli Institute for Theoretical Physics in Santa Barbara, California, and supported in part by the National Science Foundation under Grant No. NSF PHY05-51164.

${ }^{1}$ L. D. Landau and E. M. Lifshitz, Fluid Mechanics, Course of Theoretical Physics Vol. 6 (Pergamon Press, Oxford, England, 1959).

${ }^{2}$ J. T. Hynes, R. Kapral, and M. Weinberg, "Molecular theory of translational diffusion: Microscopic generalization of the normal velocity boundary condition,” J. Chem. Phys. 70(3), 1456 (1979).

${ }^{3}$ J. R. Schmidt and J. L. Skinner, "Hydrodynamic boundary conditions, the Stokes-Einstein law, and long-time tails in the Brownian limit," J. Chem. Phys. 119, 8062 (2003).
${ }^{4}$ D. Bedeaux and P. Mazur, "Renormalization of the diffusion coefficient in a fluctuating fluid III. Diffusion of a Brownian particle with finite size," Physica A: Stat. Mech. Appl. 80, 189-202 (1975).

${ }^{5}$ T. Keyes and I. Oppenheim, "Bilinear hydrodynamics and the StokesEinstein law," Phys. Rev. A 8, 937-949 (1973).

${ }^{6}$ B. J. Alder and W. E. Alley, "Generalized hydrodynamics," Phys. Today 37(1), 56 (1984).

${ }^{7}$ P. J. Atzberger, "Velocity correlations of a thermally fluctuating Brownian particle: A novel model of the hydrodynamic coupling," Phys. Lett. A 351(4-5), 225-230 (2006).

${ }^{8}$ X. Bian, S. Litvinov, R. Qian, M. Ellero, and N. A. Adams, "Multiscale modeling of particle in suspension with smoothed dissipative particle dynamics," Phys. Fluids 24(1), 012002 (2012).

${ }^{9}$ A. Vázquez-Quesada, M. Ellero, and P. Español, "Consistent scaling of thermal fluctuations in smoothed dissipative particle dynamics," J. Chem. Phys. 130, 034901 (2009)

${ }^{10}$ A. Donev, A. L. Garcia, and B. J. Alder, "Stochastic hard-sphere dynamics for hydrodynamics of non-ideal fluids," Phys. Rev. Lett. 101, 075902 (2008).

${ }^{11}$ C.-C. Huang, G. Gompper, and R. G. Winkler, "Hydrodynamic correlations in multiparticle collision dynamics fluids," Phys. Rev. E 86, 056711 (2012).

${ }^{12}$ R. Kapral, "Multiparticle collision dynamics: Simulation of complex systems on mesoscales," Adv. Chem. Phys. 140, 89 (2008).

${ }^{13}$ G. Gompper, T. Ihle, D. Kroll, and R. Winkler, "Multi-Particle collision dynamics: A particle-based mesoscale simulation approach to the hydrodynamics of complex fluids," Advanced Computer Simulation Approaches for Soft Matter Sciences III (Springer, 2009), pp. 1-87.

${ }^{14}$ B. Dünweg and A. J. C. Ladd, "Lattice Boltzmann simulations of soft matter systems," Advanced Computer Simulation for Soft Matter Sciences III (Springer, 2009), pp. 89-166.

${ }^{15}$ P. J. Atzberger, P. R. Kramer, and C. S. Peskin, "A stochastic immersed boundary method for fluid-structure dynamics at microscopic length scales," J. Comput. Phys. 224, 1255-1292 (2007).

${ }^{16}$ P. J. Atzberger, "Stochastic Eulerian-Lagrangian methods for fluidstructure interactions with thermal fluctuations," J. Comput. Phys. 230, 2821-2837 (2011).

${ }^{17}$ F. Balboa Usabiaga, R. Delgado-Buscalioni, B. E. Griffith, and A. Donev, "Inertial coupling method for particles in an incompressible fluctuating fluid," Comput. Methods Appl. Mech. Eng. (in press); preprint e-print arXiv:1212.6427.

${ }^{18}$ T. T. Pham, U. D. Schiller, J. R. Prakash, and B. Dünweg, "Implicit and explicit solvent models for the simulation of a single polymer chain in solution: Lattice Boltzmann versus Brownian dynamics," J. Chem. Phys. 131, 164114 (2009).

${ }^{19}$ A. J. C. Ladd, R. Kekre, and J. E. Butler, "Comparison of the static and dynamic properties of a semiflexible polymer using lattice Boltzmann and Brownian-dynamics simulations," Phys. Rev. E 80(3), 036704 (2009).

${ }^{20}$ J. C. Lewis, "On the einstein-stokes diffusion coefficient for Brownian motion in two dimensions," Phys. Lett. A 44(4), 245-246 (1973).

${ }^{21}$ A. Donev, A. L. Garcia, A. de la Fuente, and J. B. Bell, "Enhancement of diffusive transport by nonequilibrium thermal fluctuations," J. Stat. Mech.: Theory Exp. (2011) P06014.

${ }^{22}$ J. M. O. De Zarate and J. V. Sengers, Hydrodynamic Fluctuations in Fluids and Fluid Mixtures (Elsevier Science Ltd, 2006).

${ }^{23}$ Song Hi Lee and R. Kapral, "Friction and diffusion of a Brownian particle in a mesoscopic solvent," J. Chem. Phys. 121, 11163 (2004).

${ }^{24}$ A. Donev, T. G. Fai, and E. Vanden-Eijnden, "Reversible diffusive mixing by thermal velocity fluctuations," preprint arXiv:1306.3158 (2013).

${ }^{25}$ G. Tabak and P. J. Atzberger, "Systematic stochastic reduction of inertial fluid-structure interactions subject to thermal fluctuations," preprint arXiv:1211.3798 (2013).

${ }^{26}$ F. Balboa Usabiaga, J. B. Bell, R. Delgado-Buscalioni, A. Donev, T. G. Fai, B. E. Griffith, and C. S. Peskin, "Staggered schemes for fluctuating hydrodynamics," SIAM J. Multiscale Model. Simul. 10(4), 1369-1408 (2012).

${ }^{27}$ C. S. Peskin, "The immersed boundary method," Acta Numerica 11, 479517 (2002).

${ }^{28} \mathrm{P}$. J. Atzberger, "A note on the correspondence of an immersed boundary method incorporating thermal fluctuations with Stokesian-Brownian dynamics," Physica D: Nonlinear Phenom. 226(2), 144-150 (2007).

${ }^{29} \mathrm{H}$. Hasimoto, "On the periodic fundamental solutions of the stokes equations and their application to viscous flow past a cubic array of spheres," J. Fluid Mech. 5(02), 317-328 (1959). 
${ }^{30}$ F. Balboa Usabiaga, I. Pagonabarraga, and R. Delgado-Buscalioni, "Inertial coupling for point particle fluctuating hydrodynamics," J. Comput. Phys. 235, 701-722 (2013).

${ }^{31}$ A. M. Roma, C. S. Peskin, and M. J. Berger, "An adaptive version of the immersed boundary method," J. Comput. Phys. 153(2), 509-534 (1999).

${ }^{32}$ E. J. Hinch, "Application of the Langevin equation to fluid suspensions," J. Fluid Mech. 72(03), 499-511 (1975).

${ }^{33}$ R. M. Jendrejack, J. J. de Pablo, and M. D. Graham, "Stochastic simulations of DNA in flow: Dynamics and the effects of hydrodynamic interactions," J. Chem. Phys. 116(17), 7752-7759 (2002).

${ }^{34}$ R. Kekre, J. E. Butler, and A. J. C. Ladd, "Comparison of lattice-Boltzmann and Brownian-dynamics simulations of polymer migration in confined flows," Phys. Rev. E 82, 011802 (2010).

${ }^{35}$ Y. Pomeau and P. Résibois, "Time dependent correlation functions and mode-mode coupling theories," Phys. Rep. 19, 63-139 (1975).

${ }^{36}$ B. J. Alder and T. E. Wainwright, "Decay of the velocity autocorrelation function," Phys. Rev. A 1(1), 18-21 (1970).

${ }^{37}$ D. Bedeaux and P. Mazur, "Renormalization of the diffusion coefficient in a fluctuating fluid I," Physica 73, 431-458 (1974).

${ }^{38} \mathrm{P}$. Mazur and D. Bedeaux, "Renormalization of the diffusion coefficient in a fluctuating fluid II," Physica 75, 79-99 (1974).

${ }^{39}$ I. A. Michaels and I. Oppenheim, "Trilinear mode effects on transport coefficients," Physica A 81(4), 522-534 (1975).

${ }^{40}$ T. E. Wainwright, B. J. Alder, and D. M. Gass, "Decay of time correlations in two dimensions," Phys. Rev. A 4(1), 233-237 (1971).

${ }^{41}$ H. H. H. Yuan and I. Oppenheim, "Transport in two dimensions. III. Self-diffusion coefficient at low densities," Physica A 90(3-4), 561-573 (1978).

${ }^{42}$ C. P. Lowe and D. Frenkel, "The super long-time decay of velocity fluctuations in a two-dimensional fluid," Physica A 220(3-4), 251-260 (1995).
${ }^{43} \mathrm{M}$. Isobe, "Long-time tail of the velocity autocorrelation function in a twodimensional moderately dense hard-disk fluid," Phys. Rev. E 77(2), 021201 (2008).

${ }^{44}$ D. M. Gass, "Enskog theory for a rigid disk fluid," J. Chem. Phys. 54(5), 1898-1902 (1971).

${ }^{45}$ R. García-Rojo, S. Luding, and J. J. Brey, "Transport coefficients for dense hard-disk systems," Phys. Rev. E 74(6), 061305 (2006).

${ }^{46}$ A. P. S. Bhalla, B. E. Griffith, N. A. Patankar, and A. Donev, "A minimallyresolved immersed boundary model for reaction-diffusion problems," J. Chem. Phys. 139, 214112 (2013); preprint arXiv:1306.3159.

${ }^{47}$ R. Kubo, "The fluctuation-dissipation theorem," Rep. Prog. Phys. 29(1), 255-284 (1966).

${ }^{48}$ C. Hijón, P. Español, E. Vanden-Eijnden, and R. Delgado-Buscalioni, "Mori-Zwanzig formalism as a practical computational tool," Faraday Discuss. 144, 301-322 (2010).

${ }^{49}$ For unbounded three-dimensional systems the Oseen tensor is $\boldsymbol{G}\left(\boldsymbol{r}^{\prime}, \boldsymbol{r}^{\prime \prime}\right)$ $=(8 \pi \boldsymbol{r})^{-1}\left(\boldsymbol{I}+r^{-2} \boldsymbol{r} \otimes \boldsymbol{r}\right)$, where $\boldsymbol{r}=\boldsymbol{r}^{\prime}-\boldsymbol{r}^{\prime \prime}$.

${ }^{50}$ Note that for truly two-dimensional systems $\rho$ has units of $\mathrm{kg} / \mathrm{m}^{2}$, unlike three dimensions where it has units $\mathrm{kg} / \mathrm{m}^{3}$. This accounts for the difference in units of viscosity $\eta$ in the Stokes-Einstein relation ( $v$ has units ${ }_{0} \mathrm{~m}^{2} / \mathrm{s}$ independent of the dimension).

${ }^{51}$ Note that freely diffusing neutrally-buoyant blobs are passive tracers since they simply follow the fluid but do not affect it.

${ }^{52}$ In our model, (6) has a non-linear coupling between fluid and particle velocities. In more traditional models the no-slip boundary condition needs to be applied on a moving surface, which is hard and not done in most models, see for example the assumptions made in Ref. 32 .

${ }^{53}$ The small variation around the average value comes because the spatial discretization is only approximately translationally invariant.

${ }^{54}$ This can be seen from the fact that the last term in (15) does not appear in (16). 